\title{
Fatores associados a condutas de enfrentamento violento entre adolescentes escolares
}

\author{
Sheila Gonçalves Câmara \\ Universidade Luterana do Brasil - Canoas, RS \\ Jorge Castellá Sarriera \\ Universidade Federal do Rio Grande do Sul \\ Mary Sandra Carlotto \\ Universidade Luterana do Brasil - Canoas, $R S$
}

\begin{abstract}
Resumo
Os dados sobre violência entre jovens são preocupantes no contexto brasileiro. Neste estudo se avaliou a prática de enfrentamentos violentos em uma amostra de 389 jovens estudantes de ensino médio de Porto Alegre-RS. O objetivo foi identificar quais variáveis, em termos de dados sócio-demográficos, comportamentos arriscados no trânsito, bem-estar psicológico e estratégias de enfrentamento constituem-se em fatores de risco a prática de enfrentamentos violentos. Verificou-se uma maior chance de envolvimento em enfrentamentos entre os sujeitos do sexo masculino que conduzem veículos automotivos sem habilitação. Quanto às estratégias de coping, as chances de envolvimento são maiores entre aqueles jovens que utilizam mais as estratégias de ação social (no sentido da organização de grupos e procura por pares) e busca de diversões relaxantes, enquanto utilizam menos as estratégias de criar ilusões e autoculpar-se como alternativas para lidar com estressores. Considerando perfil de risco encontrado, faz-se necessária uma reflexão acerca do contexto social da violência e a necessidade de políticas públicas de prevenção da violência entre jovens.
\end{abstract}

Palavras-chave: adolescência; enfrentamento violento; comportamento de risco

\begin{abstract}
Risk factors associated with violent confrontation behavior among student adolescents. Violence data between youths are worrying in the Brazilian context. This study examined the violent confrontation behavior in a sample of 389 high school students of Porto Alegre-RS. The aim was to identify which variables, in terms of socio-demographic data, risky behaviors in traffic, psychological well-being and coping strategies constitutes in risk factors to violent confrontation. It was verified a bigger chance of involvement in violent confrontations between the subjects of the male sex that drive automotive vehicles without licence. Regarding to coping strategies the chances of involvement are bigger between those youths that present a bigger utilization of coping strategies of social action (in the sense of the organization of groups and seeks by pair) and seek for relaxing fun, as a minor utilization of illusions creating and self-guilty coping strategies as alternatives for deal with stressing situations. Considering this risky profile, it is necessary a reflection about the social context of violence and the need of preventive public politics to the violence between youths.
\end{abstract}

Keywords: adolescence; violent confrontation; risky behavior

A violência tem originado diversos problemas relacionados à saúde em países em desenvolvimento. $\mathrm{O}$ impacto social e sanitário é notável, uma vez que seu crescimento assume uma função linear em termos de tempo (Soares, 2006) e suas repercussões extrapolam o nível individual, atingindo a grupos populacionais (Jorge, 2002; Peres, 2002).

Atos violentos podem ser descritos como agressões dirigidas a outros, podendo ser caráter verbal, emocional ou de constrangimento (Williams-Evans \& Myers, 2004), enquanto que violência consiste na imposição de danos a alguma pessoa ou mesmo a objetos de sua propriedade que simbolizem a pessoa ou sejam seus meios de subsistência, através de atos de agressão destrutiva (Gil-Verona et al., 2002).

O conceito de violência é bastante amplo e, por vezes, controverso, porém sua forma de representação é sempre comportamental. Em termos sociais, os eventos violentos costumam ser compreendidos desde uma perspectiva individual ou isolada, e a noção de que ações violentas também podem ser 
impetradas por classes e nações, fica relegada a um segundo plano (Minayo \& Souza, 1997).

Estatisticamente, em nível mundial, os jovens despontam como o grupo social mais afetado pela violência (Center for Disease Control and Prevention, 2007). Na comparação entre as sociedades estruturalmente violentas, devido à precariedade no campo social e econômico, e países com melhores índices de qualidade de vida, as taxas de mortalidade por causas externas de jovens do sexo masculino podem ser consideradas um importante indicador. De acordo com Jorge (1998), no ano de 1995, para cada jovem morto no Canadá, Itália ou França, morriam praticamente dois jovens brasileiros.

Os estudos que abordam o tema da violência entre jovens nos Estados Unidos referem um expressivo crescimento nas últimas duas décadas (Aspy et al, 2004; Williams-Evans \& Myers, 2004). No Brasil, os índices de violência também tiveram um importante incremento, especialmente a partir da década de 90 (Instituto Brasileiro de Geografia e Estatística, 2003). De acordo com o relatório técnico do Banco Mundial (2007), Jovens em situação de risco no Brasil, a mortalidade entre os jovens devida a causas violentas cresceu de 128 para 137 pessoas em cada 100.000, comparando-se os anos de 1980 e 2002. Em 2002, entre cada 100.000 jovens, 72 morreram por causas violentas.

Não apenas as vítimas de ações violentas são jovens, como também são, geralmente, jovens os responsáveis pela violência na realidade brasileira. De acordo com Soares (2004), os jovens de 15 a 24 anos, de sexo masculino, pobres e negros, constituem as maiores vítimas de violência no Brasil. O perfil encontrado, ademais de revelar o cenário social subjacente, alerta para a relação entre violência e qualidade de vida no Brasil, de maneira que o agravo não pode ser pensado como restrito a um grupo específico, mas como uma dinâmica social.

Nesse sentido, embora exista relação entre violência e pobreza, não é possível estabelecer uma relação estrita de causalidade (Jorge, 2002). Embora as ações violentas cometidas por jovens sejam mais prevalentes entre grupos populacionais de classe sócio-econômica mais baixa (Cardia \& Schiffer, 2002), não se restringem a esse âmbito, conforme se pode verificar através da mídia e do no dia-a-dia de cidades tanto grandes como de pequeno porte, onde jovens de classe média ou alta despontam como agressores (Waiselfisz, 1998; Waiselfisz, Xavier, Maciel, \& Barbosa, 2004).

Além das ações facilmente identificadas como violentas, outros eventos, como os acidentes, também devem ser considerados na avaliação da violência como um problema de saúde pública. Dentre estes, ressaltam-se os acidentes de trânsito, que vêm atingindo contundentemente aos jovens, seja como vítimas ou como causadores (Departamento Nacional de Trânsito, 2007; Organización Mundial de la Salud, 2004; Organización Panamericana de la Salud - OPAS, 2007; Secco, 2007).

Assim como os acidentes de trânsito, muitas vezes, não são considerados dentro do enquadre da violência, também, em determinados grupos populacionais, certas ações violentas configuram um padrão habitual de relacionamento. Contrastando com os dados epidemiológicos, para muitos jovens, atos como brigas e agressões não são considerados violentos, especialmente se ocorrem em resposta a alguma provocação ou estímulo anterior, o que é mais usual entre os jovens do sexo masculino, ou se servem para manter o status social no grupo de pares (Williams-Evans \& Myers, 2004).

Com relação ao contexto, em sua especificidade, Arnett (1992) aponta que a assunção de risco estaria ligada aos contextos de socialização, como as normas apropriadas para o desempenho de papéis relacionados também ao gênero, bem como outras restrições culturais. Em termos de gênero, no entanto, além da socialização (Sarti, Barbosa, \& Suarez, 2006), faz-se importante considerar também os aspectos psicobiológicos (Gil-Verona et al., 2002). Nesse sentido, os atos violentos costumam ser mais freqüentemente impetrados por homens (OPAS, 2003).

Quanto aos processos de socialização, o grupo de pares, em conjunto com a família, a escola, as organizações de tempo livre e os meios de comunicação, representam instâncias transmissoras de valores, crenças e atitudes que vão se conformando em um estilo de vida (Balaguer, Castillo, \& Pastor, 2002). Na etapa da adolescência, para compreender como este estilo de vida pode ser mais ou menos saudável, é importante recorrer ao conceito de padrões comportamentais. Estes dizem respeito a formas recorrentes de comportamento que se executam de forma estruturada e que podem ser compreendidos como hábito quando constituem o modo regular de responder a diferentes situações (Rodríguez Marín \& García, 1995). Dessa forma, as alternativas para se lidar com situações potencialmente estressoras, o que se denomina estratégias de coping, variam de acordo com aspectos de personalidade, experiências de vida, contexto e momento específico em que tais situações ocorrem (Lazarus \& Folkman, 1984).

Frente aos estressores, de acordo com Folkman e Lazarus (1980), o individuo recorre aos recursos pessoais (habilidades de coping aprendidas no e do ambiente) e os sócio-ecológicos de coping (condições e recursos do ambiente em interação com o indivíduo), a partir dos quais se dá a seleção de estratégias, de acordo com a avaliação da relação custo-benefício. As estratégias de coping, podem ser focalizadas no problema, quando se direcionam a encontrar a origem da situação na tentativa de solucioná-la, ou na emoção, quando os esforços são dirigidos a uma busca de alívio da situação aversiva. Quanto à orientação do indivíduo frente ao problema, as estratégias de coping são avaliadas em termos aproximativos ou evitativos (Folkman \& Lazarus,1980), bem como comportamentais ou cognitivos (Moos \& Schaeler, citados por Blalock \& Joiner, 2000). Ainda que sejam vinculadas a uma situação específica com suas repercussões, a utilização de determinadas estratégias parece revelar padrões habituais relacionados ao estilo do indivíduo (Wills, 1997).

Conforme os estudos de Caffray e Schneider (2000), os adolescentes que tinham maior experiência em comportamentos de risco desenvolviam mais estratégias cognitivas para minimizar o pensamento acerca das conseqüências negativas conhecidas do comportamento. Estes achados indicam que a experiência prévia em uma conduta anterior propicia atitudes mais favoráveis à realização de comportamentos arriscados. Em termos de coping, é usual a utilização de recursos de evitação comportamental, posto que, frente a situações desagradáveis, que ocasionam tensão emocional, ações agressivas desviam do foco perturbador 
original e proporcionam o alívio da sensação aversiva através da descarga da agressividade (Hagglund, Haley, Reveille, \& Alarcón, 1989; Keefe, Brown, Wallston, \& Caldwell, 1989). Este é um comportamento que, embora possa ser considerado como funcional em termos cognitivos e emocionais, em curto prazo, tem seus custos em termos de bem-estar psicológico em longo prazo, repercutindo física e/ou psicologicamente em prejuízos pessoais ou a terceiros.

De acordo com as estatísticas que contemplam o alto índice de danos oriundos de ações violentas cometidas por jovens e também por eles sofridas (Jorge, Gawryszewski, \& Latorre, 1997), o presente estudo visou identificar variáveis referentes a características sócio-demográficas, experiência em outros comportamentos arriscados e estratégias de coping que podem constituir fatores de risco para enfrentamentos violentos entre adolescentes escolares. O objetivo último foi proporcionar uma reflexão sobre o fenômeno, subsidiando ações e programas preventivos que sejam eficazes no que se refere aos enfrentamentos violentos entre jovens.

\section{Método}

\section{Amostra}

A amostra foi composta por 389 jovens estudantes do terceiro ano do ensino médio das redes de ensino estadual e privada do município de Porto Alegre-RS, com idades entre 15 e 24 anos ( $M$ $=17,3 ; D P=1,15)$. Quanto à distribuição por sexo, $41,4 \%$ eram do sexo masculino e $58,6 \%$ do sexo feminino. Dos jovens, $60,7 \%$ estudavam em escolas públicas e 39,3\% em escolas privadas e a classe social percebida era predominantemente a classe média $(69,9 \%)$ em ambas as redes.

A seleção da amostra foi realizada conforme as seguintes etapas: (a) identificação das escolas de ensino médio, públicas e privadas, do município, de acordo com os dados da Secretaria Estadual da Educação do Rio Grande do Sul: 261 escolas estaduais e 351 escolas privadas; para cada rede de ensino foram excluídas as escolas que não ofereciam ensino médio, as escolas especiais, as técnicas e as que ofereciam ensino supletivo, o que resultou em um total de 51 escolas estaduais e 52 escolas privadas; (b) o município foi mapeado de acordo com as dez zonas eleitorais que o compõem; seguindo o critério de que, em cada zona, fossem encontradas, pelo menos, uma escola pública (estadual) e uma privada que oferecessem ensino médio, chegouse a uma divisão do município em sete regiões, também definidas por critérios sócio-econômicos e de densidade populacional; (c) as 14 escolas foram sorteadas de forma aleatória sistemática (em cada região e conforme status: pública ou privada); (d) em cada escola foi sorteada aleatoriamente uma turma de $3^{\circ}$ ano do ensino médio em turno diurno.

\section{Instrumentos}

Para avaliar as variáveis sócio-demográficas, de risco no trânsito e enfrentamentos violentos nos últimos seis meses, foi elaborado um questionário com perguntas criadas a partir da literatura referente a comportamentos de risco na juventude, visando obter as informações específicas concernentes a essa investigação (Martín González, Martínez García, López Martínez, Martín López, \& Martín Carrasco, 1997).

Para avaliar as estratégias de coping, foi utilizada a Escala de Afrontamento para Adolescentes (ACS, Frydenberg \& Lewis, 1997), adaptada por Câmara, Sarriera e Remor (2002) em cujo estudo a escala alcançou um Coeficiente Alfa de 0,85. O inventário é composto de 80 itens a serem respondidos de acordo com a freqüência de utilização das estratégias através de escala Likert de 5 pontos, sendo que, quanto maior o escore do indivíduo em cada fator, maior é a utilização da estratégia. São abarcados 18 fatores que espelham as estratégias de enfrentamento utilizadas pelos jovens: Apoio social, Resolução de problemas, Obtenção de êxito, Preocupação, Busca de relações pessoais íntimas, Busca de pertença, Esperança e antecipação de saídas positivas, Estratégia de falta de coping, Redução da tensão, Ação social, Ignorar o problema, Autoculpar-se, Reserva, Busca de apoio espiritual, Fixar-se no positivo, Busca de ajuda profissional, Busca de diversões relaxantes e Distração física.

\section{Procedimentos}

Foram realizados, inicialmente, contatos com escolas de Porto Alegre que ofereciam ensino médio, públicas e privadas, a fim de obter autorização para a realização da pesquisa entre seus alunos. Aos jovens foram esclarecidos os objetivos da pesquisa e os procedimentos éticos, tendo sido feito o uso do Termo de Consentimento Livre e Esclarecido (TCLE), que foi assinado pelos jovens maiores de idade ou, no caso dos menores, por seus responsáveis legais. O encaminhamento do TCLE para os menores de idade foi feito no dia anterior à aplicação, que ocorreu nas salas de aula, com um tempo médio de 30 minutos e sem a presença do professor.

Os dados foram submetidos a análise descritiva de caráter exploratório, a fim de avaliar, no banco de dados, casos omissos, identificação de extremos e distribuição das variáveis; e análise multivariada (Análise de Regressão Logística Binária), com a qual se pretendeu identificar, dentre as variáveis em estudo, os fatores de risco, para a variável desfecho comportamento de enfrentamento violento nos últimos seis meses.

\section{Resultados}

Inicialmente, foram analisadas as freqüências dos comportamentos de enfrentamento violento físico dentre os jovens da amostra. Verificou-se que 199 (51,2\%) participantes tiveram algum enfrentamento violento nos últimos seis meses. Com relação aos tipos de enfrentamento violento apresentados, foram identificados como mais usuais os confrontos com amigos (52,2\%); com pessoas com idéias políticas diferentes $(32,7 \%)$; com torcedores do time de futebol contrário (24,5\%), com grupos rivais $(18,5 \%)$, com vizinhos $(12,6 \%)$, na saída de festas $(12,1 \%)$ e com marginais $(11,3 \%)$. Ao ser analisada a forma de realização do comportamento, identificou-se que 55,3\% dos participantes realizaram em grupo, enquanto $44,7 \%$, individualmente.

O modelo obtido através da regressão logística multivariada alcançou uma capacidade explicativa de $64,3 \%$, demonstrando associação estatisticamente significativa de enfrentamentos 
violentos com sexo, condução de veículos automotores sem habilitação e as estratégias de coping de autoculpar-se, buscar diversões relaxantes, criar ilusões e ação social (Tabela 1).
De acordo com os resultados encontrados, os respondentes do sexo masculino têm o dobro de chances de envolverem-se em enfrentamentos violentos, em comparação com as participantes

Tabela 1

Fatores de risco para enfrentamento violento (estimativas e significância dos coeficientes). Porto Alegre, Brasil, 2004 ( $\mathrm{N}=389)$

\begin{tabular}{lrrrcc}
\hline \multicolumn{1}{c}{ Variáveis independentes } & $B$ & Wald & $g l$ & $R C(95 \% \mathrm{IC})$ & $p$ \\
\hline Sexo & $-0,692$ & 8,03 & 1 & $0,50(0,31-0,80)$ & 0,004 \\
Condução de veículo sem habilitação & 0,794 & 15,35 & 1 & $2,21(1,48-3,28)$ & 0,000 \\
ACS - Criar ilusões & $-0,099$ & 8,00 & 1 & $0,90(0,84-0,97)$ & 0,004 \\
ACS - Ação social & 0,094 & 4,50 & 1 & $1,09(1,00-1,19)$ & 0,033 \\
ACS - Autoculpar-se & $-0,121$ & 8,89 & 1 & $0,88(0,81-0,95)$ & 0,002 \\
ACS - Buscar diversões relaxantes & 0,157 & 7,72 & 1 & $1,16(1,04-1,30)$ & 0,005 \\
\hline
\end{tabular}

do sexo feminino. Da mesma forma, aqueles que conduzem veículos sem habilitação têm duas vezes mais chances de envolvimento frente aos que não apresentam esse tipo de conduta. No que tange ao uso das estratégias de ação social e busca por diversões relaxantes, verifica-se que os jovens que utilizam mais as referidas estratégias de coping apresentam mais chances de realizarem enfrentamentos violentos. Por outro lado, os enfrentamentos violentos são menos comuns entre os participantes que fazem menor uso das estratégias de coping de criar ilusões e autoculpar-se.

\section{Discussão}

O tema dos enfrentamentos violentos entre jovens tem se apresentado de difícil acesso para os pesquisadores, de maneira geral. Em grandes amostras de jovens, os enfrentamentos violentos costumam ser identificados em um pequeno número de jovens. Dessa forma, alguns pesquisadores defendem a idéia de que um entendimento mais efetivo acerca dos enfrentamentos violentos só pode ser realizado através de sub-amostras de sujeitos que assumem realizar estes comportamentos (Martín González et al., 1997).

$\mathrm{Na}$ literatura sobre o tema observa-se que, embora o tema da violência juvenil seja amplamente abordado, são poucos os estudos empíricos no Brasil que avaliem fatores predisponentes presentes da vida atual de jovens que ainda não apresentem problemas legais em relação à conduta. Observa-se uma tendência em avaliar os dados referentes à violência juvenil em termos unicamente epidemiológicos, por um lado, ou de discussão acerca de aspectos sociais envolvidos no tema, por outro. Dessa forma, não foram identificados dados de pesquisas similares na realidade brasileira que permitissem realizar comparações.

Os resultados revelaram que metade dos jovens da amostra havia tido episódios de enfrentamentos violentos físicos nos seis meses anteriores à pesquisa. Dentre estes, os enfrentamentos com amigos, com pessoas com idéias políticas diferentes (possivelmente, pessoas de maior proximidade) e com vizinhos, podem indicar uma situação considerada pontual, percebida como reversível pelo jovem, ou mesmo compartilhada entre seus pares dentro de um contexto violento (Balaguer et al., 2002; Jorge, 2002; Yunes, 2001).

O contexto violento, no entanto, pode ser explicativo apenas em termos macro-estruturais (Soares, 2006), posto que a investigação foi desenvolvida com jovens de diferentes classes sociais, o que foi operacionalizado por meio da escolha de escolas públicas e privadas que representavam diferentes estratos econômicos. E, nesse sentido, a prevalência de enfrentamentos com torcedores de time de futebol contrário, com grupos rivais, na saída de festas e com marginais amplia a compreensão acerca da magnitude do fenômeno entre jovens (OPAS, 2003). Algumas explicações podem ser dadas pela Psicologia dos Grupos, no caso dos enfrentamentos com torcedores de time contrário, mas tais explicações retirariam do cenário os fatos, cada vez mais freqüentes, de jovens de classe média e alta que se envolvem em situações de violência, beirando ou adentrando o campo da crueldade.

Ao serem avaliados os fatores de risco para o comportamento de enfrentamentos violentos, um fator de relevância nos estudos e dados epidemiológicos atuais (Banco Mundial, 2007) foi excluído a priori, qual seja, a escolaridade, posto que todos os jovens estavam finalizando o último ano do ensino médio. A escola costuma ser considerada como um fator de proteção para comportamentos de risco e delinqüência juvenil (OPAS, 2003) e, de fato, os dados sobre jovens em situação de risco no Brasil revelam associação entre baixa escolaridade e situação de risco (Banco Mundial, 2007). No entanto, ao ser enfocada uma amostra de jovens com escolarização básica praticamente concluída, e ainda inseridos no contexto escolar, identifica-se uma prevalência preocupante de condutas de enfrentamentos violentos.

Esses resultados vão ao encontro dos estudos sobre violência no contexto escolar (bullying), que revelam a gravidade a que podem chegar estes comportamentos de assédio e agressão, mesmo entre crianças (Debarbieux, 2001). Dessa forma, a 
violência parece estar instalada desde muito cedo na vida de um indivíduo, desde o contexto familiar, considerando a violência doméstica, até o escolar (Maldonado \& Williams, 2005), tendo como cenário uma sociedade incapaz de suprir as necessidades básicas, educativas e humanizadoras para seus indivíduos (Cardia \& Schiffer, 2002; Fernandes, 2004; Jorge, 2002, Ribolla \& Fiamenghi, 2007).

Os resultados sobre respondentes envolvidos em enfrentamentos violentos demonstram um perfil bastante definido: jovens do sexo masculino, que apresentam conduta de desconsideração pelas normas vigentes, como é o caso da condução de veículos sem habilitação. Em meio a um contexto sócio-cultural que confere pouco valor às normas sociais e pautase apenas pelo sistema punitivo (Peres, 2002), que é ineficaz, instaura-se a crença, muitas vezes sensata, de que condutas ilegais não trazem maiores repercussões negativas. Essa crença não é apenas um aspecto da etapa da juventude, mas uma crença partilhada em um âmbito maior, no qual pais, familiares e sistema escolar configuram-se como transmissores diretos da representação social de valores da sociedade brasileira (Camargo, Santos, Argôllo, Fernandes, \& Beserra, 2002).

As pautas sociais são ainda mais restritivas quando se trata do tema dos papéis de gênero, nos quais os atributos masculinos são relacionados a condutas de valentia que, não raramente, incorporam condutas agressivas. Os papéis de gênero, aliados a aspectos psicobiológicos (Byrner, Miller, \& Schafer, 1999), que conferem maior impulsividade e força física aos homens, fazem com que o uso da violência seja considerado natural e, até mesmo, estimulável, especialmente entre grupos cujas normas são mais rígidas (Wilson \& Daly, 1985).

Os fatores de risco para enfrentamentos violentos também estão atrelados às estratégias utilizadas pelos jovens para fazerem frente às situações estressantes que se lhes ocorrem. Tais estratégias são desenvolvidas ao longo do desenvolvimento, de acordo com as possibilidades de socialização disponíveis, os valores e normas sociais vigentes no contexto e as situações de vida em conjunção com variáveis pessoais (Folkman, Lazarus, Dunkel-Schetter, DeLongis, \& Gruen, 1986).

Em termos de coping, é sempre bastante complexo falar de estratégias positivas ou negativas, pois isso requer uma análise aprofundada dos fatores imbricados no uso de cada uma delas (Lazarus \& Folkman, 1984). No caso das estratégias que podem representar fatores de risco para a conduta de enfrentamentos violentos identificam-se as estratégias de criar ilusões, ação social, autoculpar-se e busca por distrações relaxantes.

No que tange à busca por distrações relaxantes, o que se pode afirmar é que é uma estratégia evitativa (Carver, Scheier, \& Weintraub, 1989), porém sua configuração pode ser bastante variada, desde a busca por esportes até o uso de substâncias químicas, como uma forma de tirar de foco o estressor e aliviar a tensão. Tal estratégia requer atividade e, no caso de representar um fator de risco para enfrentamentos violentos, faz pensar que o alívio da tensão está vinculado a atividades agressivas, que, frente a situações frustrantes, geradoras de emoções de descontentamento e raiva, consistem em uma alternativa de alívio tensional (Caffray \& Schneider, 2000). Ao ser vinculada a estratégia de busca de distrações relaxantes com a ação social, forma-se um panorama que pode ser interpretado como a busca de apoio por parte dos pares para a realização de tal atividade, especialmente considerando os itens que avaliavam esta estratégia no instrumento, ou seja, itens que agrupavam questões de identidade grupal (Frydenberg \& Lewis, 1997).

Ambas as estratégias de coping, busca de distrações relaxantes e ação social, de acordo com esta compreensão, podem ser interpretadas como formas pontuais e atuais na vida do jovem, de lidar com os estressores. No entanto, as estratégias de criar ilusões e autoculpar-se remetem a todo um sistema de desenvolvimento e aprendizagem no decorrer da vida do indivíduo (Lazarus \& Folkman, 1984). Criar ilusões vincula-se a uma riqueza simbólica que, por sua vez, está atrelada a possibilidades reais de vida e futuro (Fernandes, 2004; Frydenberg \& Lewis, 1997). Embora a adolescência seja um período fértil em termos do campo simbólico (Balaguer et al., 2002; Camargo et al., 2002), é preciso considerar que a possibilidade de imaginar um futuro positivo acaba sendo relegada a um segundo plano tanto para os jovens que estão impedidos de pensar em oportunidades por sua situação sócio-econômica (Soares, 2004), como para aqueles que, sem impedimentos sócio-econômicos maiores, encontramse aborrecidos em um cotidiano que lhes proporciona poucas possibilidades criativas (Pavía, Gerlero, \& Apendino, 1995; Sarriera, Zandonai, Hermel, Mousquer, \& Coelho, 2007).

A estratégia de autoculpar-se também pode ser compreendida tanto em termos negativos como positivos. Negativamente, considerando o desenvolvimento psicológico, o uso extremado dessa estratégia pode repercutir em baixa auto-eficácia (Bandura $\&$ Wood, 1989). No entanto, em se tratando de enfrentamentos violentos, verificou-se que os jovens que utilizam mais a estratégia de autoculpabilização têm menos chances de envolverse em enfrentamentos violentos. Isto porque se pode pensar na culpa como uma forma de "arrependimento antecipado", conforme Caffray e Schneider (2000).

A culpa, nesse caso, torna-se estreitamente vinculada à violência ou a comportamentos agressivos como um conseqüente. Estaria constituída pelas normas e valores construídos por meio das experiências de vida dos jovens em seus espaços de socialização (Balaguer et al., 2002). O arrependimento antecipado pode ser compreendido como um fator de dissonância entre as atitudes prevalentes na vida do jovem e as situações ocasionais, de violência, com as quais se depara (Caffray \& Schneider, 2000).

Dentro de um enfoque psicossocial considera-se que a adoção de um tipo ou outro de estilo de vida vai depender do resultado das interações do jovem com suas circunstâncias peculiares de vida. $\mathrm{O}$ que não se pode precisar é se o jovem tem clareza acerca do risco atrelado aos comportamentos violentos e se essa escolha é, ou não, voluntária, considerando os padrões culturais enquanto construções sociais (Benthin, Slovic, \& Severson, 1993; Cohn, Macfarlane, Yanez, \& Imai, 1995; Weinstein; 1987). Ambientes mais saudáveis proporcionarão um estilo de vida mais saudável, ao passo que condições de vida desfavoráveis podem contribuir para estilos de vida menos saudáveis (Costa \& López, 2000). Para o indivíduo, estilos de vida que incorporem violência conduzem a conseqüências para a saúde ou para a vida, interferindo nos domínios de saúde, papéis 
sociais, desenvolvimento pessoal e preparação para a vida adulta (Balaguer et al., 2002).

No âmbito social, o perfil de incorporação de comportamentos violentos ao estilo de vida deve ser considerado de acordo com sua magnitude enquanto problema de saúde pública, uma vez que a violência juvenil afeta família, amigos e comunidades. Traz conseqüências negativas não somente em termos de mortes, agravos e incapacitação física permanente, mas em termos da qualidade de vida de uma sociedade (OPAS, 2003).

Conforme a OPAS (2002), a violência caracteriza-se por uma multiplicidade de fatores inter-relacionados, como é o caso dos aspectos biológicos, psicológicos sociais e ambientais. Dessa forma, não existe uma ação única ou simples para sua solução. As abordagens em torno do tema devem contemplar intervenções centradas nos jovens e em seus relacionamentos, assim como nas comunidades e diversos setores da sociedade.

Uma limitação deste estudo é o fato de a maioria dos jovens que participou da pesquisa (95\%) estudar de dia, de maneira que o estudo se restringe a jovens que estão no ensino regular e que, pelo turno de estudo, não trabalhavam ou trabalhavam apenas em meio turno. Certamente, essa população se diferencia dos jovens que estudam à noite em termos de contingências de vida e até mesmo de média etária.

Cabe salientar que foram encontrados aspectos que podem ser aprofundados através de questionamentos aos jovens e que podem permitir análises mais aprofundadas sobre este fenômeno. Nesse sentido, sugere-se o desenvolvimento de pesquisas com abordagem qualitativa junto a esta população, assim como estudos longitudinais que permitam uma melhor compreensão acerca dos antecedentes e possíveis desdobramentos da violência juvenil.

\section{Referências}

Arnett, J. J. (1992). Reckless behavior in adolescence: a developmental perspective. Developmental Review, 12, 339-373.

Aspy, C. B., Oman, R. F., Vesely, S. K, McLeroy, K., Rodine. S., \& Marshall. L. (2004). Adolescent violence: the protective effects of youth assets. Journal of Counseling \& Development, 82, 268-276.

Balaguer, I. , Castillo, I., \& Pastor, Y. (2002). Los estilos de vida relacionados con la salud en la adolescencia temprana (pp. 5-26). In I. Balaguer (Org.), Estilos de vida en la adolescencia. Valencia: Promolibro.

Banco Mundial (2007). Jovens em situação de risco no Brasil - Volume II: Relatório Técnico. Brasília: Autor.

Bandura, A., \& Wood, R. (1989). Impact of conceptions of ability on selfregulatory mechanisms and complex decision making. Journal of Personality and Social Psychology, 56(3), 407-415.

Benthin, A., Slovic, P. E., \& Severson, H. (1993). A psychometric study of adolescent risk perception. Journal of Adolescence, 16, 153-168.

Blalock, J. A., \& Joiner, T. E. (2000). Interaction of cognitive avoidance coping and stress in predicting depression/anxienty. Cognitive Therapy and Research, 24(1), 47-65.

Byrnes, J. P., Miller, D. C., \& Schafer, W. D. (1999). Gender differences in risk taking: a meta-analysis. Psychological Bulletin, 125, 367-383.

Caffray, C. M., \& Schneider, S. L. (2000). Why do they do it? Affective motivators in adolescents' decisions to participate in risk behaviors. Cognition and Emotion, 14, 543-576.

Câmara, S. G., Sarriera, J. C., \& Remor, E. A. (2002). Análise fatorial da Escala de Afrontamento para Adolescentes (ACS) em uma amostra de jovens de
Porto Alegre. Aletheia, 16, 15-32.

Camargo, C. L., Santos, N. O. D., Argôllo, S. L. S., Fernandes, S. L. S. A., \& Beserra, M. A. (2002). Violência no contexto familiar e escolar. In M. C. O. Costa \& R. P. Souza (Orgs.). Adolescência: aspectos clínicos e psicossociais (pp. 432-438). Porto Alegre: Artes Médicas.

Cardia, N., \& Schiffer, S. (2002). Violência e desigualdade social. Ciência e Cultura, 54(1), 25-31.

Carver, C. S., Scheier, M. F., \& Weintraub, J. K. (1989). Assessing coping strategies: a theoretically based approach. Journal of Personality and Social Psychology, 56, 267-283.

Center for Disease Control and Prevention - CDC. (2007). National health interview survey, Unites States, 2006. Morbidity \& Mortality Weekly Report, 56, 37.

Cohn, L., MacFarlane, S., Yanez, C., \& Imai, W. K. (1995). Risk-perception: differences between adolescents and adults. Health Psychology, 14(3), 217-222.

Costa, M., \& López, E. (2000). Educación para la salud: una estrategia para cambiar los estilos de vida. Madri: Pirámide.

Debarbieux, E. (2001, abril). Violencia escolar: un problema mundial. El Correo de UNESCO, disponível em http://www.unesco.org/courier/2001_04/sp/ education.htm; acessado em 27 de março de 2005.

Departamento Nacional de Trânsito - DENATRAN (2007). O jovem e o trânsito. II Seminário de Educação e Segurança no Trânsito. Disponível em http:// www.denatran.gov.br/seminario/jovem/II_seminario.htm; acessado em 19 de setembro de 2007

Fernandes, R. C. (2004). Segurança para viver: propostas para uma política de redução da violência entre adolescentes e jovens. In R. Novaes \& P. Vannuchi (Orgs.), Juventude e sociedade: trabalho, educação, cultura e participação (pp. 260-274). São Paulo: Fundação Perseu Abramo.

Folkman, S., \& Lazarus, R. S. (1980). An analysis of coping in a middle-aged community sample. Journal of Health and Social Behavior, 21, 219-239.

Folkman, S., Lazarus, R. S., Dunkel-Schetter, C., DeLongis, A., \& Gruen, R. J. (1986). Dynamics of a stressful encounter: cognitive appraisal, coping, and encounter outcomes. Journal of Personality and Social Psychology, 50(5), 992-1003.

Frydenberg, E., \& Lewis, R. (1997). Escalas de afrontamiento para adolescentes: Manual. Madri: Publicaciones de Psicología Aplicada.

Gil-Verona, J. A., Pastor, J. F., De Paz, F., Barbosa, M., Macías, A., Maniega, M. A., Rami-González, L., Boget, T., \& Picornell, I. (2002). Psicobiología de las conductas agresivas. Anales de Psicología, 18, 293-303.

Hagglund, K. J., Haley, W. E., Reveille, J. D., \& Alarcón, G. S. (1989). Predicting individual differences in pain and functional impairment among patients with rheumatoid artritis. Artritis and Rheumatism, 32, 851-858.

Instituto Brasileiro de Geografia e Estatística - IBGE (2003). Síntese de indicadores sociais 2003. Disponível em http://www.ibge.gov.br; acessado em 26 de setembro de 2004

Jorge, M. H. P. M. (1998). Como morrem nossos jovens. In Comissão Nacional de População e Desenvolvimento (Org.), Jovens acontecendo na trilha das políticas públicas (pp. 209-289). Brasília: Autor.

Jorge, M. H. P. M. (2002). Violência como problema de saúde pública. Ciência e Cultura, 54(1), 52-53.

Jorge, M. H. P. M, Gawryszewski, V. P., \& Latorre, M. R. D. O (1997). I - Análise dos dados de mortalidade. Revista de Saúde Pública, 31(4 suppl.), 5-25.

Keefe, F. J., Brown, G. K., Wallston, K. A., \& Caldwell, D. S. (1989). Coping with rheumatoid arthritis pain: catastrophizing as a maladaptative strategy. Pain, 37, 51-56.

Lazarus, R. S., \& Folkman, S. (1984). Stress, appraisal, and coping. Nova York: Springer.

Maldonado, D. P. A., \& Williams, L. C. A. (2005). O comportamento agressivo de crianças do sexo masculino na escola e sua relação com a violência doméstica. Psicologia em Estudo, 10(3), 353-362.

Martín González, A., Martínez García, J. M., López Martínez, J., Martín López, M. J., \& Martín Carrasco, J. M. (1997). Comportamientos de riesgo: 
violencia, prácticas sexuales de riesgo y consumo de drogas ilegales en la juventud. Madri: Entinema.

Minayo, M. C. S., \& Souza, E. R. (1997). Violência e saúde como um campo interdisciplinar e de ação coletiva. História, Ciências, Saúde-Manguinhos, 4(3), 513-531.

Organización Mundial de la Salud - OMS (2004). Informe mundial sobre prevención de los traumatismos causados por el tránsito: resumen. Genebra: Autor.

Organización Panamericana de la Salud - OPAS (2002). Informe mundial sobre la violencia y la salud. Washington, D.C.: Autor.

Organización Panamericana de la Salud - OPAS (2003). Informe mundial sobre la violencia y la salud. Washington, D.C.: Autor.

Organización Panamericana de la Salud - OPAS (2007). Primera semana mundial de las Naciones Unidas sobre la seguridad vial. Manual para la organización de actividades. Washington, D.C.: Autor.

Pavía, V., Gerlero, J., \& Apendino, J. (1995). Adolescencia, grupo y tiempo libre. Buenos Aires: Humanitas.

Peres, M. F. T. (2002).Prevenção e controle: oposição ou complementaridade para a redução da violência? Ciência e Cultura, 54(1), 54-55.

Ribolla, M. B., \& Fiamenghi Jr., G. A. (2007). Adolescentes na escola: representações sociais sobre violencia. Psicologia Escolar e Educacional, 11(1), 111-121.

Rodríguez Marín, J., \& García, J. A. (1995). Estilo de vida y salud. In J. M. Latorre (Org.), Ciencias psicossociales aplicadas II (pp. 25-34). Madri: Síntesis.

Sarriera, J. C., Zandonai, Z. K. G., Hermel, J. S., Mousquer, P. N., \& Coelho, R. P. S. (2007). A relação entre pesquisadores e escolas públicas: um diálogo a partir do tempo livre. Psicologia e Sociedade, 19(1), 85-89.

Sarti, C. A., Barbosa, R. M., \& Suarez, M. M. (2006). Violência e gênero: vítimas demarcadas. Physis: Revista de Saúde Coletiva, 16(2), 167-183.

Secco, F. S. (2007). Jovens vidas em risco: o que temos a ver com isso? Disponível em http://www.vivamais.rs.gov.br/upload/artigo/artigo6.pdf; acessado em 30 de setembro de 2007.

Soares, G. A. D. (2006). Whose death is it, anyway? Ciência e Saúde Coletiva, 11(2), 273-275.

Soares, L. E. (2004). Juventude e violência no Brasil contemporâneo. In R. Novaes \& P. Vannuchi (Orgs.), Juventude e sociedade: trabalho, educação, cultura e participação (pp. 130-159). São Paulo: Fundação Perseu Abramo/ Instituto Cidadania.

Waiselfisz, J. J. (1998). Juventude, violência e cidadania: os jovens de Brasília. São Paulo: Cortez.

Waiselfisz, J. J., Xavier, R., Maciel, M., \& Barbosa, P. D. (2004). Relatório de desenvolvimento juvenil 2003. Brasília: UNESCO.

Weinstein, M. D. (1987). Unrealistic optimism about susceptibility to health problems: conclusions from a community-wide sample. Journal of Behavioral Medicine, 10(5), 481-500.

Williams-Evans, S. A., \& Myers, J. S. (2004). Adolescent violence. The ABNF (Association of Black Nursing Faculty in Higher Education) Journal, 15(2), 31-34

Wills, T. A. (1997). Modes and families of coping: an analysis of downward comparison in the structure of other cognitive and behavioral mechanisms. New Jersey: Erlbaum.

Wilson, M., \& Daly, M. (1985). Competitiveness, risk taking, and violence: the young male syndrome. Ethnology and Sociobiology, 6, 59-73.

Yunes, J. (2001). Epidemiologia da violência. In M. C. Oliveira (Org.), Demografia da exclusão social: temas e abordagens (pp. 145-165). Campinas: Hucitec.

Sheila Gonçalves Câmara, doutora em Psicologia pela Pontifícia Universidade Católica do Rio Grande do Sul, é professora no Curso de Psicologia e Programa de Pós-Graduação em Saúde Coletiva da Universidade Luterana do Brasil/Canoas, RS. Endereço para correspondência: Rua Edmundo Gardolinski, 70; Porto Alegre, RS; CEP: 90480-130. E-mail: scamara@via-rs.net

Jorge Castellá Sarriera, doutor em Psicologia Social pela Universidade Autônoma de Madrid, é professor no Programa de Pós-Graduação em Psicologia da Universidade Federal do Rio Grande do Sul. E-mail: sarriera@terra.com.br Mary Sandra Carlotto, doutora em Psicologia Social pela Universidade de Santiago de Compostela, é professora no Curso de Psicologia da Universidade Luterana do Brasil/Canoas, RS. E-mail: mscarlotto@ gmail.com 